Original paper

\title{
Simple non-invasive markers for early diagnosis and determination of the severity of liver diseases
}

\author{
Monika Gudowska', Alicja Wrona', Ewa Gruszewska', Anatol Panasiuk², Bogdan Cylwik³ , Magdalena Swiderska², \\ Robert Filisiak², Maciej Szmitkowski', Lech Chrostek' \\ 'Department of Biochemical Diagnostics, Medical University of Bialystok, Poland \\ 2Department of Infectious Diseases and Hepatology, Medical University of Bialystok, Poland \\ ${ }^{3}$ Department of Pediatric Laboratory Diagnostics, Medical University of Bialystok, Poland
}

\begin{abstract}
Aim of the study: The aim of the study was to evaluate the effect and severity of liver diseases of different etiologies on the values of three non-invasive fibrosis markers.

Material and methods: Serum samples from 65 patients with alcoholic cirrhosis, 31 with non-alcoholic cirrhosis and 32 with toxic hepatitis, were tested. Cirrhotic patients were classified according to the Child-Pugh scale. The age-platelet (AP) index, HUl score and Fibro Q index were calculated using the specific formulas.

Results: The values of all tested scores were significantly higher in controls than in patients with liver diseases and were significantly different between liver diseases. The patients with alcoholic and non-alcoholic cirrhosis had higher values of the AP index, HUl score and Fibro Q index than patients with toxic hepatitis. $\mathrm{HUl}$ and Fibro Q scores appeared to vary according to the severity of liver damage and were higher in Child-Pugh class $C$ than in classes A and B.

Conclusions: We conclude that all tested scores based on liver function tests are good markers for non-invasive diagnosis of liver damage. Additionally, HUI and Fibro Q scores reflect the severity of liver cirrhosis.
\end{abstract}

Key words: liver cirrhosis, toxic hepatitis, non-invasive markers.

\section{Address for correspondence}

Monika Gudowska, Department of Biochemical Diagnostics, Medical University of Bialystok, 15A Waszyngtona St., 15-269 Białystok, Poland, phone/fax: +48 8574685 85, e-mail: monika.gudowska@umb.edu.pl

\section{Introduction}

Liver cirrhosis is the final stage of fibrosis characterized by architectural distortion and the formation of regenerative nodules. Cirrhosis, resulting from various chronic liver diseases, is an important problem of public health in highly developed countries, being one of the most common causes of death in Europe. Literature data suggest that about $0.1 \%$ of the European population is affected by liver cirrhosis, which corresponds to 26 new cases per 100,000 population annually [1-3].

Apart from the ultrasound, computed tomography (CT), magnetic resonance imaging (MRI) and liver biopsy, the diagnosis of liver diseases can be based on the results of biochemical tests $[4,5]$. Liver function tests, such as proteins, liver enzymes, bilirubin, albumin or platelet count in the blood, help to detect inflammation and liver damage. In particular, a decrease in the synthesis of anti-coagulant proteins can result in an increased level of the international normalized ratio (INR) [6]. In addition, a very common hematological abnormality in patients with liver diseases is thrombocytopenia (platelet [PLT] count $<150 \times 10^{9} / 1$ ). The drop in platelet count may occur due to decreased platelet production in the bone marrow (e.g. caused by viruses or alcoholic etiology of liver disease), increased platelet destruction through increased shear stress or decreased activity of thrombopoietin $[7,8]$. The death of hepatocytes and inflammation can also result in elevated serum aminotransferases activity. Alanine ami- 
notransferase (ALT) is found mainly in the cytosol of hepatocytes, and is released into the blood during liver damage [9]. By contrast, aspartate aminotransferase (AST) is present not only in the liver, but is also found in several organs including heart and muscles. As AST level itself is not a good predictor of liver damage, it is usually expressed as the AST/ALT ratio $[9,10]$.

The main plasma protein produced by the liver is albumin, and the gradual destruction of liver tissue over time may lead to reduced albumin synthesis. A decreased level of albumin is a prognostic factor for advanced liver diseases [11]. Another important function of the liver is bilirubin excretion into the bile. If bilirubin cannot be efficiently removed from the liver, a high level of bilirubin accumulates in the blood $[12,13]$.

The aim of this study was to evaluate the changes in the values describing liver functions based on three non-invasive indirect markers of liver disease age-platelet (AP) index, HUI score and Fibro Q index - depending on the liver disease etiologies or severity. These indexes are based on the determination of PLT, bilirubin, albumin, AST, ALT and INR adjusted to age and body mass index (BMI).

\section{Material and methods}

\section{Human subjects}

The experimental group consisted of 128 patients (70 men and 58 women, age between 24 and 88 years) consecutively admitted to the Department of Infectious Diseases and Hepatology, Medical University of Bialystok. The diagnosis was based on the results of a biochemical liver panel (PLT count, mean corpuscular volume [MCV], INR, AST, ALT, $\gamma$-glutamyltransferase [GGT], albumin and bilirubin) and other clinical data (signs, symptoms, physical examination results, and abdominal ultrasound or computed tomography scan of the abdomen). To confirm the diagnosis of hepatitis $\mathrm{C}$ virus (HCV), an anti-HCV test was performed. According to the clinical diagnosis, the liver disease

Table 1. Definition of age-platelet (AP) index

\begin{tabular}{ccc}
\hline Score & Age (years) & PLT (10 $/$ I) \\
\hline 0 & $<30$ & $\geqslant 225$ \\
\hline 1 & $30-39$ & $200-224$ \\
\hline 2 & $40-49$ & $175-199$ \\
\hline 3 & $50-59$ & $150-174$ \\
\hline 4 & $60-69$ & $125-149$ \\
\hline 5 & $\geqslant 70$ & $<125$ \\
\hline
\end{tabular}

was classified as: alcoholic cirrhosis (AC) - 65 patients, non-alcoholic cirrhosis (NAC) - 31 patients and toxic hepatitis (HT) - 32 patients. Non-alcoholic cirrhosis was caused by chronic hepatitis $\mathrm{C}-15$ patients, chronic hepatitis $\mathrm{B}-1$, autoimmune hepatitis - 1, primary biliary cirrhosis -5 and by undefined factors -9 . Toxic hepatitis is an inflammation of the liver, and in our study it was caused by alcohol in 23 patients and by drugs in 9 patients. The severity of liver cirrhosis was evaluated by the Child-Pugh scale (class A - 31 subjects, class $B-37$ and class $C-28$ ). All patients were interviewed regarding their use of alcohol.

The control group (C) consisted of 30 healthy people (17 males and 13 females). All (healthy and sick) subjects gave their informed consent to participate in the study. This study was in accordance with the Helsinki Declaration and was approved by the Bioethical Committee at the Medical University of Bialystok.

\section{Blood sampling}

Fasting blood samples were taken by vein puncture after hospital admission and before treatment. The sera were separated by centrifugation and stored at $-86^{\circ} \mathrm{C}$ until assayed. Besides serum, a part of each blood sample was collected into tubes containing 3.8\% liquid sodium citrate and EDTA-2.

AST, ALT and bilirubin were determined on an Architect c8000 (Abbott Laboratories, Abbott Park, USA), and albumin was determined on an Image 800 analyzer (Beckman Coulter Diagnostics, USA). Prothrombin time (PT) was determined on an STA Compact Max analyzer (Diagnostica Stago, France), and PLT count was determined on a Sysmex XS-800i (Sysmex Corporation, Singapore).

\section{Calculations}

AP index was calculated based on the following formula [14]:

$$
\mathrm{AP} \text { index }=\text { age }+\operatorname{PLT}\left(\frac{10^{9}}{1}\right)
$$

The AP index has a range of possible values from 0 to 10 (Table 1). The total possible score derives from two parameters: age and PLT. Different points are given and added together according to the values of these parameters.

HUI score [15] and Fibro Q index [16] were calculated using the formulas:

HUI score $=3.148+0.167 \times B M I+0.088 \times$ bilirubin $-0.151 \times$ albumin $-0.019 \times \operatorname{PLT}\left(\frac{10^{9}}{l}\right)$ 


$$
\text { Fibro } \mathrm{Q}=\frac{10 \times \text { age } \times \operatorname{AST}\left(\frac{\mathrm{U}}{l}\right) \times \operatorname{INR}}{\operatorname{PLT}\left(\frac{10^{9}}{l}\right) \times \operatorname{ALT}\left(\frac{\mathrm{U}}{l}\right)}
$$

\section{Statistical analysis}

The differences between tested and control groups were evaluated using the Mann-Whitney $U$ test. To test the hypothesis about the differences between liver diseases, the ANOVA rank Kruskal-Wallis test was performed. We considered $p$ values less than 0.05 statistically significant. The diagnostic performance of each test was calculated as sensitivity, specificity, positive predictive value (PPV), negative predictive value (NPV) and diagnostic accuracy (ACC). The area under the receiver operating characteristic curve (AUC) was used to calculate the diagnostic performance of all algorithms.

\section{Results}

The values of scores calculated from results of noninvasive blood tests are presented in Table 2 . The values of the AP index, HUI score and Fibro Q index were significantly higher in alcoholic cirrhosis (AC) and non-alcoholic cirrhosis (NAC) groups in comparison to the control group ( $p<0.001$ for all comparisons). Also, the values of AP, HUI and Fibro Q scores were higher in toxic hepatitis (HT) patients compared to the controls $(p<0.001, p=0.037, p=0.018$, respectively). The analysis of variance revealed that liver diseases affects the AP, HUI and Fibro Q scores ( $p<0.001, p=0.012, p<0.001$, respectively). The patients with alcoholic cirrhosis had higher values of AP index, HUI score and Fibro Q index than patients with toxic hepatitis $(p<0.001, p=0.014, p<0.001$, respectively). In addition, AP index, HUI score and Fibro Q index were significantly higher in non-alcoholic cirrhosis in comparison with toxic hepatitis patients $(p<0.001$, $p=0.045, p=0.018$, respectively), but there was no significant difference between non-alcoholic cirrhosis and alcoholic cirrhosis patients $(p=0.269, p=0.912$, $p=0.175$, respectively). ANOVA rank Kruskal-Wallis analysis showed that Child-Pugh stage does not have an impact on the AP index $(p=0.334)$, but has an impact on the HUI and Fibro Q scores ( $p<0.001$ for both). Further analysis showed that their values were higher in class $C$ patients than in class $A$ ( $p<0.001$ for both). Additionally, HUI score and Fibro Q index levels were higher in class $\mathrm{C}$ than in class $\mathrm{B}$ ( $p<0.001$ for both).

Diagnostic usefulness of non-invasive markers is presented in Table 3. Age-platelet index has the highest ability to detect alcoholic cirrhosis and toxic hepatitis (sensitivity of $98.2 \%$ and $100 \%$, respectively), but the HUI score has the highest ability to exclude both of these diseases (specificity of 100\%). Additionally, the HUI score exhibited the highest sensitivity (100\%) and specificity (100\%) in non-alcoholic cirrhosis. The highest diagnostic power (AUC) in the detection of alcoholic cirrhosis and toxic hepatitis can be attributed to the AP index (AUC \pm SE; AC: $0.973 \pm 0.015, \mathrm{HT}$ : $0.855 \pm 0.056$ ), but the HUI score has the highest diagnostic power in non-alcoholic cirrhosis (AUC $\pm \mathrm{SE}$ : $1.00 \pm 0.00)$. In all of the diseases tested, the positive predictive value (PPV) of some of the indexes equals $100 \%$. Also, PPV of the AP index in non-alcoholic cirrhosis equals $100 \%$. These high values resulted from the lack of false-negative results.

\section{Discussion}

Prognosis in chronic liver diseases depends on the stage and severity of liver fibrosis [17]. The gold standard for the diagnosis of liver diseases is still a liver

Table 2. Results of laboratory tests in controls (C) and patients with liver diseases and according to Child-Pugh score

\begin{tabular}{lccc}
\hline Group & AP index & HUI & Fibro Q \\
\hline Controls $(n=30)$ & $1.35 \pm 1.57$ & $2.09 \pm 0.04$ & $1.84 \pm 1.03$ \\
\hline $\mathrm{AC}(n=65)$ & $7.08 \pm 1.92^{*}, \mathrm{c}$ & $5.18 \pm 2.42^{*}, \mathrm{c}$ & $29.78 \pm 14.40^{*}, \mathrm{c}$ \\
\hline $\mathrm{NAC}(n=31)$ & $7.84 \pm 1.88^{*}, \mathrm{c}$ & $5.36 \pm 1.34^{*}, \mathrm{c}$ & $14.17 \pm 12.62^{*}, \mathrm{c}$ \\
\hline $\mathrm{HT}(n=32)$ & $4.48 \pm 2.37^{*}, \mathrm{a,b}$ & $3.99 \pm 2.50^{*, \mathrm{a}, \mathrm{b}}$ & $8.93 \pm 7.56^{*, a, b}$ \\
\hline Child-Pugh A $(n=31)$ & $6.87 \pm 2.46$ & $4.39 \pm 2.55^{\mathrm{c}}$ & $11.18 \pm 10.30^{\mathrm{C}}$ \\
\hline Child-Pugh B $(n=37)$ & $6.92 \pm 2.03$ & $4.95 \pm 1.98^{\mathrm{C}}$ & $21.71 \pm 23.98^{\mathrm{C}}$ \\
\hline Child-Pugh C $(n=28)$ & $7.79 \pm 1.29$ & $6.72 \pm 1.50^{\mathrm{A}, \mathrm{B}}$ & $47.21 \pm 39.52^{\mathrm{A}, \mathrm{B}}$ \\
\hline
\end{tabular}

Data are mean \pm standard deviation

*Significant differences in comparison to controls. Significant differences in comparison to (ANOVA rank Kruskal-Wallis): $A C-a, N A C-b, H T-c, C h i l d-P u g h A-A, C h i l d-P u g h$ B - B, Child-Pugh C-C 
Table 3. Diagnostic value of age-platelet (AP), HUI and Fibro Q scores in liver diseases

\begin{tabular}{lccccccc}
\hline Liver disease & Cut-off (from ROC) & Sensitivity [\%] & Specificity [\%] & ACC [\%] & PPV [\%] & NPV [\%] & AUC \pm SE \\
\hline AP index & \multicolumn{7}{l}{} \\
\hline AC & 3.0 & 98.2 & 70.0 & 90.8 & 90.2 & 93.3 & $0.973 \pm 0.015$ \\
\hline NAC & 5.0 & 93.1 & 100.0 & 95.9 & 100.0 & 90.9 & $0.986 \pm 0.013$ \\
\hline HT & 1.0 & 100.0 & 45.0 & 73.8 & 66.7 & 100.0 & $0.855 \pm 0.056$ \\
\hline HUl score & & & & & & & \\
\hline AC & 2.6 & 92.9 & 100.0 & 94.7 & 100.0 & 83.3 & $0.929 \pm 0.034$ \\
\hline NAC & 2.12 & 100.0 & 100.0 & 100.0 & 100.0 & 100.0 & $1.0 \pm 0.0$ \\
\hline HT & 2.81 & 52.6 & 100.0 & 76.9 & 100.0 & 69.0 & $0.526 \pm 0.115$ \\
\hline Fibro Q index & & & & & & 85.7 & $0.965 \pm 0.021$ \\
\hline AC & 3.06 & 94.4 & 90.0 & 93.2 & 96.2 & 96.4 & $0.959 \pm 0.025$ \\
\hline NAC & 4.26 & 88.9 & 95.0 & 91.5 & 96.0 & 86.0 \\
\hline HT & 3.33 & 38.1 & 95.0 & 65.9 & 88.9 & 59.4 & $0.6 \pm 0.092$ \\
\hline
\end{tabular}

AC - alcoholic cirrhosis, NAC - non-alcoholic cirrhosis, HT - toxic hepatitis, ACC - diagnostic accuracy, PPV - positive predictive value, NPV - negative predictive value, AUC - area under the ROC curve, SE - standard error

biopsy. The examination of liver tissue samples provides a good estimate of liver histological structure and the degree of liver fibrosis. Liver biopsy is used to obtain about $1 / 50000$ of the liver mass. Because it is a very small part of this organ, biopsy is associated with a high rate of sampling error [18-20]. Also, the big disadvantage of liver biopsy is its invasiveness and complications in up to $5.0 \%$ of patients (e.g. bleeding, pain, bile peritonitis, right kidney puncture and death) [21]. In addition, there are many contraindications to perform liver biopsy, such as: being an uncooperative patient, bacterial cholangitis, abnormal coagulation indices, thrombocytopenia, ascites, cystic lesions or obesity [20]. Therefore, it is necessary to find early diagnostic markers of liver fibrosis that can be measured in the blood. Circulating markers of liver disease progression are divided into class I - markers directly representing the degree of liver fibrogenesis - and class II - biomarkers which are a mathematical combination of biochemical and cytological markers [17]. In this study we aimed to determine the diagnostic value of three markers included in mathematical algorithms: the AP index, HUI score and Fibro Q index. Non-invasive fibrosis detection is important as liver damage leads to impairment of liver function.

The AP index, based on platelet count and age, is inexpensive, easy, and rapid to calculate. It was invented by Poynard and Bedossa, who wanted to find a simple diagnostic index in HCV patients classified according to the presence of necroinflammatory lesions and fibrosis of the liver [22]. Poynard and Bedossa demon- strated that platelet count and age combined in a simple algorithm correlated with the presence of fibrosis and histological activity of the disease. According to these data the value $\geq 6$ of the AP index had a specificity of $93 \%$, sensitivity of $52 \%$ and area under the ROC curve of $0.690 \pm 0.085$. The authors assumed that an AP score of 6 or more could circumvent the need of liver biopsy, but its negative predictive value was not high enough to prevent a liver biopsy in patients with a lower score. In other studies, the diagnostic power measured according to Ishak and Kondell scores as the area under the ROC curve (AUC) in HCV patients with $\geq$ F3 hepatic fibrosis reached a value between 0.57 and 0.61 . At the cutoff of 4 or greater the AP index exhibited a sensitivity of $80 \%$ and specificity of $45 \%$, but at the cutoff of 6 or greater the AP index had lower sensitivity (30\%) and higher specificity (77\%) for the diagnosis of histological fibrosis with scores of $\geq 3$ [23]. Our study revealed that the AP index has excellent efficiency for the diagnosis of alcoholic and nonalcoholic cirrhosis (AUC: 0.973, 0.929, respectively). On the other hand, our study indicates that the AP index is unable to differentiate between the stages of liver cirrhosis according to the Child-Pugh scale. In summary, AP index calculation can be used to detect chronic hepatitis $\mathrm{C}$, toxic hepatitis and cirrhosis and reflects the degree of liver cirrhosis. The AP index has very good diagnostic accuracy for predicting cirrhosis, but it is not useful to evaluate the severity of liver cirrhosis according to the Child-Pugh scale. 
The next noninvasive marker is the HUI score based on the body mass index and three routine laboratory tests: albumin, bilirubin and platelet count. This predictive model was developed by Hui et al., who wanted to develop a non-invasive model which could potentially decrease the need for liver biopsy in some patients with chronic hepatitis B [24]. They proved that this algorithm was accurate in predicting absence of significant fibrosis. In our study the specificity and positive predictive value were the same for alcoholic cirrhosis, non-alcoholic cirrhosis and toxic hepatitis $(100 \%, 100 \%$, respectively). This PPV resulted from the lack of false-negative results. There were significant differences between liver diseases. HUI scores were significantly higher in alcoholic and non-alcoholic cirrhosis than in toxic hepatitis, but there were no differences between cirrhosis of different etiologies. Similar results were obtained by Sebastiani et al., who observed high PPV (> 96.3\%) for HUI in advanced fibrosis with a cut-off of 0.15 , but its diagnostic value was quite low and reached the value of 0.71 AUC (95\% CI: 0.56-0.86). Therefore, the HUI score has a potential to be used for the detection of liver diseases, excluding significant fibrosis, evaluation of fibrosis degree and differentiation of cirrhosis of different etiologies from toxic hepatitis.

The Fibro Q score can predict alcoholic cirrhosis as well as non-alcoholic cirrhosis with $98.2 \%$ and $88.9 \%$ sensitivity, respectively, but the ability to detect toxic hepatitis is low (sensitivity: $38.1 \%$ and AUC: 0.6$)$. We also found that Fibro Q has different values according to the severity of liver cirrhosis. The Fibro Q was approximately 5 times higher in class $\mathrm{C}$ patients compared with class $A$ and 2 times higher in class $C$ compared with class B. Our data are consistent with the study of Hsieh et al. They showed that Fibro Q is an easy tool to evaluate significant fibrosis in patients with chronic viral hepatitis. Among the chronic hepatitis patients who had significant fibrosis/cirrhosis, only $3.45 \%$ of patients had Fibro Q scores of 0.6 or lower. Additionally, in patients with Fibro Q scores of 1.6 or greater, $92.9 \%$ had significant fibrosis, and only $7.1 \%$ of patients without significant fibrosis were incorrectly classified. Hsieh et al. also compared the accuracy of Fibro Q with other non-invasive algorithms - AST-toplatelet ratio index (APRI), and AST/ALT ratio (AAR) - and demonstrated that Fibro Q is better than APRI, and equal to AAR, as a biomarker for predicting significant fibrosis [25]. In summary, our study and that of Hsieh et al. indicate that the Fibro Q value can quickly evaluate the progression of chronic liver diseases. It is a good marker to differentiate cirrhotic patients from those with toxic hepatitis. The data suggest that the value of this index differs depending on the intensity of the disease process and its chronicity.

\section{Conclusions}

We conclude that the values of simple algorithms are changed in liver diseases and may depend on the severity of liver cirrhosis. Our results indicate the high usefulness of these noninvasive scores for detection and exclusion of liver diseases, especially cirrhosis.

\section{Disclosure}

The authors report no conflict of interest.

\section{References}

1. Blachier M, Leleu H, Peck-Radosavljevic M, et al. The burden of liver disease in Europe: a review of available epidemiological data. J Hepatol 2013; 58: 593-608.

2. Starr SP, Raines D. Cirrhosis: diagnosis, management, and prevention. Am Fam Physician 2011; 84: 1353-1359.

3. Tsochatzis EA, Bosch J, Burroughs AK. Liver cirrhosis. Lancet 2014; 383: 1749-1761.

4. Chalasani N, Younossi Z, Lavine JE, et al. The diagnosis and management of non-alcoholic fatty liver disease: practice Guideline by the American Association for the Study of Liver Diseases, American College of Gastroenterology, and the American Gastroenterological Association. Hepatology 2012; 55: 2005-2023.

5. Festi D, Schiumerini R, Marzi L, et al. Review article: the diagnosis of non-alcoholic fatty liver disease-availability and accuracy of non-invasive methods. Aliment Pharmacol Ther 2013; 37: 392-400.

6. Harding DJ, Perera MT, Chen F, et al. Portal vein thrombosis in cirrhosis: Controversies and latest developments. World J Gastroenterol 2015; 21: 6769-6784.

7. Mitchell O, Feldman DM, Diakow M, et al. The pathophysiology of thrombocytopenia in chronic liver disease. Hepat Med 2016; 8: 39-50.

8. Afdhal N, McHutchison J, Brown R, et al. Thrombocytopenia associated with chronic liver disease. J Hepatol 2008; 48: 10001007.

9. Kim WR, Flamm SL, Di Bisceglie AM, et al. Serum activity of alanine aminotransferase (ALT) as an indicator of health and disease. Hepatology 2008; 47: 1363-1370.

10. Oh RC, Hustead TR. Causes and evaluation of mildly elevated liver transaminase levels. Am Fam Physician 2011; 84: 1003-1008.

11. Oettl K, Stadlbauer V, Petter F, et al. Oxidative damage of albumin in advanced liver disease. Biochim Biophys Acta 2008; 1782: 469-473.

12. Fleischner G, Arias IM. Recent advances in bilirubin formation, transport, metabolism and excretion. Am J Med 1970; 49: 576-589.

13. Chowdhury NR, Chowdhury JR. Disorders of Bilirubin Metabolism. In: The Liver: Biology and Pathobiology. $5^{\text {th }}$ ed. John Wiley \& Sons, Ltd., Chichester 2009.

14. Lackner C, Struber G, Liegl B, et al. Comparison and validation of simple noninvasive tests for prediction of fibrosis in chronic hepatitis C. Hepatology 2005; 41: 1376-1382.

15. Hui AY, Chan HL, Wong VW, et al. Identification of chronic hepatitis B patients without significant liver fibrosis by a simple 
noninvasive predictive model. Am J Gastroenterol 2005; 100: 616-623.

16. Hsieh YY, Tung SY, Lee K, et al. Routine blood tests to predict liver fibrosis in chronic hepatitis C. World J Gastroenterol 2012; 18: 746-753.

17. Jarcuska P, Bruha R, Horvath G, et al. Evaluation of hepatic fibrosis - access to non-invasive methods, national practice/guidelines in Central Europe. Clin Exp Hepatol 2016; 1: 12-15.

18. Bota S, Sirli R, Sporea I, et al. A new scoring system for prediction of fibrosis in chronic hepatitis C. Hepat Mon 2011; 11: 548-555.

19. Colli A, Colucci A, Paggi S, et al. Accuracy of a predictive model for severe hepatic fibrosis or cirrhosis in chronic hepatitis C. World J Gastroenterol 2005; 11: 7318-7322.

20. Rockey DC, Caldwell SH, Godman ZD, et al. Liver biopsy. Hepatology 2009; 49: 1017-1044.

21. Cross TJ, Calvaruso V, Maimone S, et al. Prospective comparison of Fibroscan, King's score and liver biopsy for the assessment of cirrhosis in chronic hepatitis C infection. J Viral Hepat 2010; 17: 546-554.

22. Poynard T, Bedossa P. Age and platelet count: a simple index for predicting the presence of histological lesions in patients with antibodies to hepatitis C virus. J Viral Hepat 1997; 4: 199-208.

23. Gökcan H, Kuzu UB, Öztaş E, et al. The predictive value of noninvasive serum markers of liver fibrosis in patients with chronic hepatitis C. Turk J Gastroenterol 2016; 27: 156-164.

24. Hui AY, Chan HL, Wong VW, et al. Identification of chronic hepatitis B patients without significant liver fibrosis by a simple noninvasive predictive model. Am J Gastroenterol 2005; 100: 616-623.

25. Hsieh YY, Tung SY, Lee IL, et al. FibroQ: an easy and useful noninvasive test for predicting liver fibrosis in patients with chronic viral hepatitis. Chang Gung Med J 2009; 32: 614-622. 